\title{
STUDY OF THE EFFECTIVENESS OF DIFFERENT KALMAN FILTERING METHODS AND SMOOTHERS IN OBJECT TRACKING BASED ON SIMULATION TESTS
}

\author{
Marcin Malinowski, Janusz Kwiecień \\ University of Technology and Life Sciences \\ Geomatics, Geodesy and Spatial Economy Department \\ Bydgoszcz, Poland.
}

\begin{abstract}
In navigation practice, there are various navigational architecture and integration strategies of measuring instruments that affect the choice of the Kalman filtering algorithm. The analysis of different methods of Kalman filtration and associated smoothers applied in object tracing was made on the grounds of simulation tests of algorithms designed and presented in this paper. EKF (Extended Kalman Filter) filter based on approximation with (jacobians) partial derivations and derivative-free filters like UKF (Unscented Kalman Filter) and CDKF (Central Difference Kalman Filter) were implemented in comparison. For each method of filtration, appropriate smoothers EKS (Extended Kalman Smoother), UKS (Unscented Kalman Smoother) and CDKS (Central Difference Kalman Smoother) were presented as well. Algorithms performance is discussed on the theoretical base and simulation results of two cases are presented.
\end{abstract}

Keywords: Kalman filtering, smoother, Extended Kalman Filter, derivativefree filtering, Central Difference Kalman Filter, Unscented Kalman Filter, object tracing

\section{Introduction}

Every modern navigation system used in terrestrial, airborne and underwater moving objects is designed to provide information about its position, velocity and acceleration as accurately as possible (Kwiecień et al., 2006). Using these data allows one to specify the position of the object in a desired coordinate system. One can theoretically assume the existence of an independent measuring device along with a mechanism that provides the solution of the navigation task on the basis of data received. This approach, however, requires the use of a device with high precision 
and reliability. If the technical implementation of such assumptions appeared to be possible, the cost of such project would be very high.

This state of affairs requires choosing other ways, such as construction of integrated navigation systems, where measuring data that come from several less accurate sensors is processed by an assumed computational algorithm. In such systems the importance of measuring devices often depends on the accuracy of the device and a model that characterizes the distribution of a measurement error.

In integrated navigation systems the most common tool for processing the measurements is the Kalman filter. In its original form it acts as an optimal algorithm for information processing in systems with a linear model of dynamics and measurement. The form of the Kalman filter function is adequate to the expectations and tasks associated with the construction of integrated navigation systems.

Except the Kalman filter and its numerous modifications: EKF (Extended Kalman Filter), UKF (Unscented Kalman Filter) and CDKF (Central Difference Kalman Filter) which compose the group of Gauss approximation filters, there are alternative forms of filtration, such as the PF (Particle Filter) group of filters based on SMC, sequential Monte Carlo methods. The first three of the above mentioned methods of filtration were used in algorithms and simulation studies presented in this paper.

Integrated navigation system can use the following architecture: uncoupled, loosely coupled, tightly coupled or deeply coupled (Christian, 2000; GrejnerBrzezińska et al., 2005; Kaniewski, 2006; Kim et al., 2003; Knight, 1999; Vorbrich, 2011).

\section{Filtering methods and smoothers}

\subsection{Extended Kalman filter EKF}

The Kalman filter in its classical form (Kalman, 1960), cannot be used in systems where the state estimation model or measurement model is nonlinear. The first chronologically proposed and implemented adaptation of the Kalman filter for nonlinear models was the linearization of the covariance matrix (Rogers, 2007). This method is called an Extended Kalman Filter, in short EKF.

In the extended version of the Kalman filter, the linearization is performed by expanding the nonlinear function in Taylor series. The first word is usually used to evolve into series. This solution found many practical applications, mainly because of the speed. However, the approximation by using the partial derivatives (jacobians) for highly nonlinear models in practice proves to be insufficient (van der Merwe and Wan and Julier, 2004). EKF algorithm for nonlinear system in dynamic model can be presented in the following form (Särkkä 2006):

$$
\begin{gathered}
\boldsymbol{x}_{k}=f\left(\boldsymbol{x}_{k-1}, k-1\right)+\boldsymbol{q}_{k-1} \\
\boldsymbol{z}_{k}=h\left(\boldsymbol{x}_{k}, k\right)+\boldsymbol{r}_{k}
\end{gathered}
$$

where:

$k$ - discrete time moment;

$\boldsymbol{x}_{k}, \boldsymbol{x}_{k-1}$ - the state vector at time $k$ and $k-1, \boldsymbol{x}_{k} \wedge \boldsymbol{x}_{k-1} \in \mathbb{R}^{n}$ respectively;

$\boldsymbol{z}_{k}$ - vector of observations, $\boldsymbol{z}_{k} \in \mathbb{R}^{p}$;

$\boldsymbol{q}_{k-1}$ - vector of random variable representing the process noise, $\boldsymbol{q}_{k-1} \sim$

$N\left(0, \boldsymbol{Q}_{k-1}\right)$; 
$\boldsymbol{r}_{k}$ - vector of random variable representing the measurement noise, $\boldsymbol{r}_{k} \sim$ $N\left(0, \boldsymbol{R}_{k}\right)$.

The task of the functions $f$ and $h$, appearing in equations (1) and (2) is description of models respectively: the state and observation. The extended version of the Kalman filter is directly derived from the classical form that is why the analogy between them is so obvious. Before activating the cyclical measuring procedure, the initialization of variables should be done.

- Prediction step: at this stage, the prediction of the state vector $\widehat{\boldsymbol{x}}_{k}^{\ominus}$ (3) and covariance matrix $\boldsymbol{P}_{k}^{\ominus}(4)$ by using the Jacobians is determined as follows (Särkkä, 2006):

$$
\begin{gathered}
\widehat{\boldsymbol{x}}_{k}^{\ominus}=f\left(\widehat{\boldsymbol{x}}_{k-1}, k-1\right) \\
\boldsymbol{P}_{k}^{\ominus}=\boldsymbol{F}_{k-1, i, j} \boldsymbol{P}_{k-1} \boldsymbol{F}_{k-1, i, j}^{T}+\boldsymbol{Q}_{k-1} \\
\boldsymbol{F}_{k-1, i, j}=\nabla f=\left.\frac{\partial f_{i}}{\partial x_{k-1, j}}\right|_{\boldsymbol{x}_{k-1}=\widehat{\boldsymbol{x}}_{k-1}}=\left[\begin{array}{ccc}
\frac{\partial f_{1}}{\partial x_{k-1,1}} & \cdots & \frac{\partial f_{1}}{\partial x_{k-1, j}} \\
\vdots & \ddots & \vdots \\
\frac{\partial f_{i}}{\partial x_{k-1,1}} & \cdots & \frac{\partial f_{i}}{\partial x_{k-1, j}}
\end{array}\right]
\end{gathered}
$$

where:

$\boldsymbol{F}_{k-1, i, j}$ - the Jacobian defined as matrix whose ij-th element is the partial derivative of the $i$-th element of vector nonlinear function $f$ relative to the $j$ element of the state vector $\mathbf{x}$ at the moment $k-1$ in closest of $\boldsymbol{x}_{k-1}=\widehat{\boldsymbol{x}}_{k-1}$; $\boldsymbol{Q}_{k-1}$ - covariance matrix of process noise.

- Correction step: At this stage, we set out with the calculation of innovation $\boldsymbol{v}_{k}$ (or observation residue) as a difference between the realization of observation $\boldsymbol{z}_{k}$ and prediction of observation $h\left(\hat{\boldsymbol{x}}_{k}^{\ominus}, k\right)$. Furthermore, we calculate the innovation covariance matrix $S_{k}$.(Särkkä, 2006)

$$
\begin{gathered}
\boldsymbol{v}_{k}=\boldsymbol{z}_{k}-h\left(\widehat{\boldsymbol{x}}_{k}^{\ominus}, k\right) \\
\boldsymbol{S}_{k}=\boldsymbol{H}_{k, i, j} \boldsymbol{P}_{k}^{\ominus} \boldsymbol{H}_{k, i, j}^{T}+\boldsymbol{R}_{k} \\
\boldsymbol{H}_{k, i, j}=\nabla h=\left.\frac{\partial h_{i}}{\partial x_{k, j}}\right|_{\boldsymbol{x}_{k}=\widehat{\boldsymbol{x}}_{k}^{\ominus}}=\left[\begin{array}{ccc}
\frac{\partial h_{1}}{\partial x_{k, 1}} & \cdots & \frac{\partial h_{1}}{\partial x_{k, j}} \\
\vdots & \ddots & \vdots \\
\frac{\partial h_{i}}{\partial x_{k, 1}} & \cdots & \frac{\partial h_{i}}{\partial x_{k, j}}
\end{array}\right]
\end{gathered}
$$

where:

$\boldsymbol{H}_{k, i, j}$ - the Jacobian defined as matrix whose ij-th element is the partial derivative of the $i$-th element of vector nonlinear function $h$ relative to the $j$-th element of state vector $\boldsymbol{x}$ at the moment $k$ in the environment of $\boldsymbol{x}_{k}=\widehat{\boldsymbol{x}}_{k}^{\ominus}$; $\boldsymbol{R}_{k}$ - covariance matrix of measurement noise. 
Then the gain matrix $\boldsymbol{K}_{k}$ is used to update the estimates of the state vector and covariance matrix from the first stage of prediction according to the following expressions (Särkkä, 2006):

$$
\begin{gathered}
\boldsymbol{K}_{k}=\boldsymbol{P}_{k}^{\ominus} \boldsymbol{H}_{k}^{T}\left(\boldsymbol{S}_{k}\right)^{-1} \\
\widehat{\boldsymbol{x}}_{k}=\widehat{\boldsymbol{x}}_{k}^{\ominus}+\boldsymbol{K}_{k} \boldsymbol{v}_{k} \\
\boldsymbol{P}_{k}=\boldsymbol{P}_{k}^{\ominus}-\boldsymbol{K}_{k} \boldsymbol{S}_{k} \boldsymbol{K}_{k}^{T}
\end{gathered}
$$

\subsection{UKF filter}

The method of approximation of the model described above allows to achieve reliable results, if the nature of the error propagation can be approximated by a linear function (jacobians). Otherwise, the ability of the function that approximates a filter may be insufficient and, in extreme cases the filter may be divergent. In addition, the determination of jacobians may be difficult or impossible.

Thus, the presented KF and UKF filters due to their nature, have some imperfections, and therefore limited scope of application. An alternative is UKF filter so called Unscented Kalman Filter (van der Merwe and Wan 2001; Konatowski et al., 2004). This is a recursive estimated filter, which reflects the nature of strongly nonlinear models well (Särkkä, 2006). Unlike the EKF, the unscented filter is not a linear model, but operates on the statistical parameters which are subjected to the nonlinear transformations of the state and observation vectors. The functioning of the UKF is an unscented transformation (in short UT), which is a method of calculating descriptive parameters of the random variable subjected to the nonlinear transformation on the assumption that it is more convenient to estimate the probability distribution rather than the nonlinear function (van der Merwe and Wan 2001). To calculate the mean and covariance of the $n$-dimensional random variable for the nonlinear transformation is necessary to determine the weight set of $2 n+1$ sigma points $\left\{\mathcal{X}_{i}, \mathcal{W}_{i}\right\}$ as the set of pairs of information about the value of the $i$-th sigma point and corresponding to its importance (weight). These points are selected to have a common base value of the mean $\overline{\boldsymbol{x}}$ and covariance matrix $\boldsymbol{P}_{\boldsymbol{x}}$. Assuming that the variable $\boldsymbol{x} \in \mathbb{R}^{n} \boldsymbol{x}$ has a Gaussian distribution $N\left(\overline{\boldsymbol{x}}, \boldsymbol{P}_{x}\right)$, its components are subjected to the transformation by the nonlinear function $\mathcal{Y}_{i}=f\left(x_{i}\right)$. This unscented transformation tends to determine the most accurate possible approximation of the probability distribution of the variable $\mathbf{y}$. The weighted unscented transformation is the basis of the filter UKF and is done in every step of the algorithm, for each step separately, i.e. prediction and correction. Therefore, the amount of computation UKF filter is much larger than for the EKF algorithm.

\subsection{CDKF filter}

Regardless of the work carried out on the development of UKF filter, two independent groups of scientists have submitted a proposal for a filter based on nonlinear function (Ito and Xiong, 2000), which does not require linearization of the model using Jacobians, and its work is based on Stirling's polynomial interpolation. This formula became the basis for the filter DDF (Divided Difference Filter) presented in and the CDF (Central Difference Filter) published in (Ito and Xiong, 2000). Both filters, though developed independently, function basically the same, hence it was decided to 
harmonize their names as the central difference filter (Central Difference Kalman Filter) in short CDKF (van der Merwe and Wan, 2001).

\subsection{Smoothing methods}

RTS (Rauch-Tung-Striebel) smoothing method (Rauch, 1965), (Nørgaard, 2000), (Shin, 2005) for the Kalman filter in brief KS (Kalman Smoother) allows smoothing the solution of the state dynamic model described by expression (1). The difference between the classical Kalman filter and its smoothing algorithm depends on the fact that, the filter run forward and takes into account only the past information, but the smoother proceeds backward in time from last epoch. Some inconvenience in forward process is the necessity of storing in every epoch predicted state vector and covariance matrix. This demand increase the need for amount of storing space.

The smoothing computation process of EKS algorithm starts with calculating prediction of the state vector $\widehat{\boldsymbol{x}}_{k+1}^{\ominus}$ (12) and state covariance matrix $\boldsymbol{P}_{k+1}^{\ominus}$ (13).

$$
\begin{gathered}
\widehat{\boldsymbol{x}}_{k+1}^{\ominus}=f\left(\widehat{\boldsymbol{x}}_{k}, \boldsymbol{q}_{k}\right) \\
\boldsymbol{P}_{k+1}^{\ominus}=\boldsymbol{F}_{k} \boldsymbol{P}_{k} \boldsymbol{F}_{k}^{T}+\boldsymbol{Q}_{k} \\
\boldsymbol{F}_{k, i, j}=\nabla f=\left.\frac{\partial f_{i}}{\partial x_{k, j}}\right|_{\boldsymbol{x}_{k}=\widehat{\boldsymbol{x}}_{k}}=\left[\begin{array}{ccc}
\frac{\partial f_{1}}{\partial x_{k, 1}} & \cdots & \frac{\partial f_{1}}{\partial x_{k, j}} \\
\vdots & \ddots & \vdots \\
\frac{\partial f_{i}}{\partial x_{k, 1}} & \cdots & \frac{\partial f_{i}}{\partial x_{k, j}}
\end{array}\right]
\end{gathered}
$$

In next step gain matrix $\boldsymbol{C}_{k}(15)$ should be calculated, then we indicate smoothed estimate of state vector $\widehat{\boldsymbol{x}}_{k}^{S}(16)$ and smoothed state covariance matrix $\boldsymbol{P}_{k}^{S}(17)$.

$$
\begin{gathered}
\boldsymbol{C}_{k}=\boldsymbol{P}_{k} \boldsymbol{A}_{k}^{T}\left(\boldsymbol{P}_{k+1}^{\ominus}\right)^{-1} \\
\widehat{\boldsymbol{x}}_{k}^{s}=\widehat{\boldsymbol{x}}_{k}+\boldsymbol{C}_{k}\left(\widehat{\boldsymbol{x}}_{k+1}^{s}-\widehat{\boldsymbol{x}}_{k+1}^{\ominus}\right) \\
\boldsymbol{P}_{k}^{s}=\boldsymbol{P}_{k}+\boldsymbol{C}_{k}\left(\boldsymbol{P}_{k+1}^{s}-\boldsymbol{P}_{k+1}^{\ominus}\right) \boldsymbol{C}_{k}^{T}
\end{gathered}
$$

The procedure presented above in the form of expressions from (10) to (15) is a smoothing algorithm in version corresponding to the Extended Kalman filter. There are also adaptation of the RTS algorithm connected with UKF and PF filters (Särkkä, 2006, 2008; Särkkä et al., 2007; Shin 2005; Shin et al., 2005).

\section{Examples of demonstration projects}

For the filtration methods and smoothers described above there were developed computer programs based on the original, modular library of estimation algorithms BEA (Bayesian Estimation Algorithms). The implementation was migrated from Matlab environment and performed in the Object Pascal language because of the need to independent computing, versatility and the ease of integration with other software and measuring devices.

The examples shown below were carried out to verify the correctness and quality of work of designed filtration methods in conditions of fitting the state dynamics and 
observation models to the actual dynamic system. Because of this, in every example the task of generator block was to provide on the input of each of the filters in the mode of matching to the actual dynamic system the observation of measuring noise parameters $r$ and the parameters of state of the system dynamics with process noise $\boldsymbol{q}$. To ensure identical conditions of simulation, the covariance matrices of procedural errors $\boldsymbol{Q}$ and of measurement errors $\boldsymbol{R}$ for the concerned system in all tested filters had the same form. At the same time, in each example shown at the beginning of the estimation process, in all the analyzed methods of filtration, the same model of initial parameters $E\left[\boldsymbol{x}_{0}\right]$ and $\boldsymbol{P}_{0}$ was adopted.

\subsection{Case 1 - The trajectory of a moving object which is observed from two positions with the measurement of directions}

The demonstration presented in this section relates to the estimation of tracking a moving object which is observed from two fixed positions $s^{1}$ and $s^{2}$ using measurement of directions $\theta_{k}^{1}$ and $\theta_{k}^{2}$ (Fig. 1).

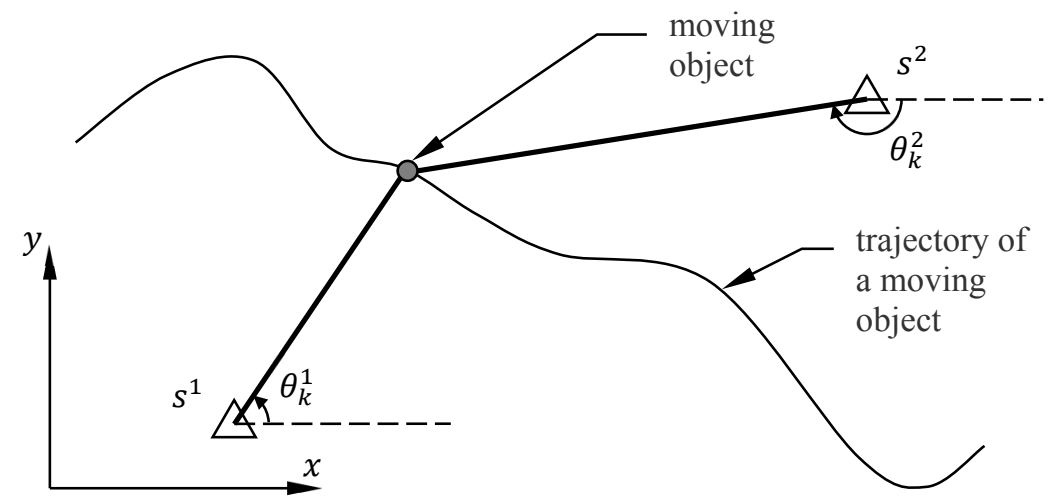

Fig.1. The trajectory of a moving object observed from two fixed positions using the two direction measurements

The state vector of the dynamics of observed object, consisted of its position and velocity in a rectangular coordinate system, was formulated as follows:

where:

$$
\boldsymbol{x}_{k}=\left[\begin{array}{llll}
x_{k} & \dot{x}_{k} & y_{k} & \dot{y}_{k}
\end{array}\right]^{T}=\left[\begin{array}{llll}
p_{k}^{x} & v_{k}^{x} & p_{k}^{y} & v_{k}^{y}
\end{array}\right]^{T}
$$

$x_{k}, y_{k}, p_{k}^{x}, p_{k}^{y}$ - rectangular coordinates of the object at the time $k$,

$\dot{x}_{k}, \dot{y}_{k}, v_{k}^{x}, v_{k}^{y}$-components of the object velocity at the time $k$.

State dynamic of the target was modeled as Continuous Wiener Process Velocity (CWPV) model, which is presented by the following expression:

$$
\frac{d \boldsymbol{x}(t)}{d t}=\left[\begin{array}{llll}
0 & 1 & 0 & 0 \\
0 & 0 & 0 & 0 \\
0 & 0 & 0 & 1 \\
0 & 0 & 0 & 0
\end{array}\right] \boldsymbol{x}(t)+\left[\begin{array}{ll}
0 & 0 \\
1 & 0 \\
0 & 0 \\
0 & 1
\end{array}\right] \boldsymbol{q}(t)
$$


and Gaussian process noise $\boldsymbol{q}(t)$ defines the spectral density matrix:

$$
\boldsymbol{Q}_{c}=\left[\begin{array}{ll}
q & 0 \\
0 & q
\end{array}\right]
$$

in which parameter $q$ describes the size of perturbations of the random variable for two velocity components of the observed object, which was determined as $q=0.1$. Discrete form of a (Taylor series) in continuous model can be written as:

$$
\boldsymbol{x}_{k}=\left[\begin{array}{cccc}
1 & \Delta t & 0 & 0 \\
0 & 1 & 0 & 0 \\
0 & 0 & 1 & \Delta t \\
0 & 0 & 0 & 1
\end{array}\right] \boldsymbol{x}_{k-1}+\boldsymbol{q}_{k-1}
$$

where the time increment $\Delta t=0.01 \mathrm{~s}$ was adopted.

Gaussian process noise $\boldsymbol{q}_{k-1}$ of the expected value $E\left[\boldsymbol{q}_{k-1}\right]=0$ and covariance matrix $\boldsymbol{Q}_{k-1}$ can be determined using the following expression:

$$
\boldsymbol{Q}_{k-1}=E\left[\boldsymbol{q}_{k-1}\left(\boldsymbol{q}_{k-1}\right)^{T}\right]=\left[\begin{array}{cccc}
\frac{1}{3} \Delta t^{3} & \frac{1}{2} \Delta t^{2} & 0 & 0 \\
\frac{1}{2} \Delta t^{2} & \Delta t & 0 & 0 \\
0 & 0 & \frac{1}{3} \Delta t^{3} & \frac{1}{2} \Delta t^{2} \\
0 & 0 & \frac{1}{2} \Delta t^{2} & \Delta t
\end{array}\right] q
$$

Observation model for two positions with coordinates $\left(s_{x}^{1}, s_{y}^{1}\right)=(-1,-2)$ and $\left(s_{x}^{2}, s_{y}^{2}\right)=(1,1)$ can be written as the following equation:

$$
\boldsymbol{z}_{k}=\nabla h\left(\boldsymbol{x}_{k}\right)+\boldsymbol{R}_{k}, \text { where } h\left(\boldsymbol{x}_{k}\right)=\left[\begin{array}{c}
\theta_{k}^{1} \\
\theta_{k}^{2}
\end{array}\right]=\left[\begin{array}{l}
\arctan \left(\frac{y_{k}-s_{y}^{1}}{x_{k}-s_{x}^{1}}\right) \\
\arctan \left(\frac{y_{k}-s_{y}^{2}}{x_{k}-s_{x}^{2}}\right)
\end{array}\right]
$$

Assuming no correlation between the measurements of direction, taking the measurement error covariance matrix $\boldsymbol{R}_{k}$ as white noise with expected value $E\left[\boldsymbol{r}_{k}\right]=0$, and assuming that the variance is $\sigma_{r}=0.05(\mathrm{rad})$, equation will have the following form: 


$$
\boldsymbol{R}_{k}=\left[\begin{array}{cc}
\sigma_{r}^{2} & 0 \\
0 & \sigma_{r}^{2}
\end{array}\right]=\left[\begin{array}{cc}
0.0025 & 0 \\
0 & 0.0025
\end{array}\right]
$$

After calculating partial derivatives, the Jacobi matrix can be written as follows:

$$
\boldsymbol{H}_{k}=\left[\begin{array}{cccc}
\frac{-\left(y_{k}-s_{y}^{1}\right)}{\left(x_{k}-s_{x}^{1}\right)^{2}+\left(y_{k}-s_{y}^{1}\right)^{2}} & 0 & \frac{x_{k}-s_{x}^{1}}{\left(x_{k}-s_{x}^{1}\right)^{2}+\left(y_{k}-s_{y}^{1}\right)^{2}} & 0 \\
\frac{-\left(y_{k}-s_{y}^{2}\right)}{\left(x_{k}-s_{x}^{2}\right)^{2}+\left(y_{k}-s_{y}^{2}\right)^{2}} & 0 & \frac{x_{k}-s_{x}^{2}}{\left(x_{k}-s_{x}^{2}\right)^{2}+\left(y_{k}-s_{y}^{2}\right)^{2}} & 0
\end{array}\right]
$$

where coordinates of positions are: $\left(s_{x}^{1}, s_{y}^{1}\right)=(-1,-2)$ and $\left(s_{x}^{2}, s_{y}^{2}\right)=(1,1)$.

To ensure identical conditions of simulation, the covariance matrices of state errors $\boldsymbol{Q}_{k-1}$ and of measurement errors $\boldsymbol{R}_{k}$ for the system under consideration in all tested filters have the same form. Similarly, at the beginning of the calculation in all analyzed filtration methods, the same state vector of Gaussian distribution $N\left(E\left[\boldsymbol{x}_{0}\right], \boldsymbol{P}_{0}\right)$ has been adopted with an initial expected value:

$$
E\left[\boldsymbol{x}_{0}\right]=\left[\begin{array}{llll}
0 & 0.5 & 0 & 0
\end{array}\right]^{T}
$$

and initial state covariance matrix:

$$
\boldsymbol{P}_{0}=\left[\begin{array}{cccc}
0.1 m^{2} & 0 & 0 & 0 \\
0 & 10(\mathrm{~m} / \mathrm{s})^{2} & 0 & 0 \\
0 & 0 & 0.1 m^{2} & 0 \\
0 & 0 & 0 & 10(\mathrm{~m} / \mathrm{s})^{2}
\end{array}\right]
$$

This notation of the matrix of covariance indicates a significant degree of confidence to the position, and much uncertainty relative to the velocity of the observed target.

$\mathrm{n}$ the generator block of the system dynamics that was used to determine the trajectory of the observed object, an initial state vector was adopted as follows:

$$
\boldsymbol{x}_{0}=\left[\begin{array}{llll}
0 & 0.5 & 0 & 0
\end{array}\right]^{T}
$$

\subsection{Case 2 - The trajectory of a moving object observed from two positions with using the direction and distance measurement}

The second demonstration issue presented in this section concerns the estimation of the track of an object from two fixed observation positions using not only the measurement of direction as in 5.1, but also of the distance (Fig. 2). 


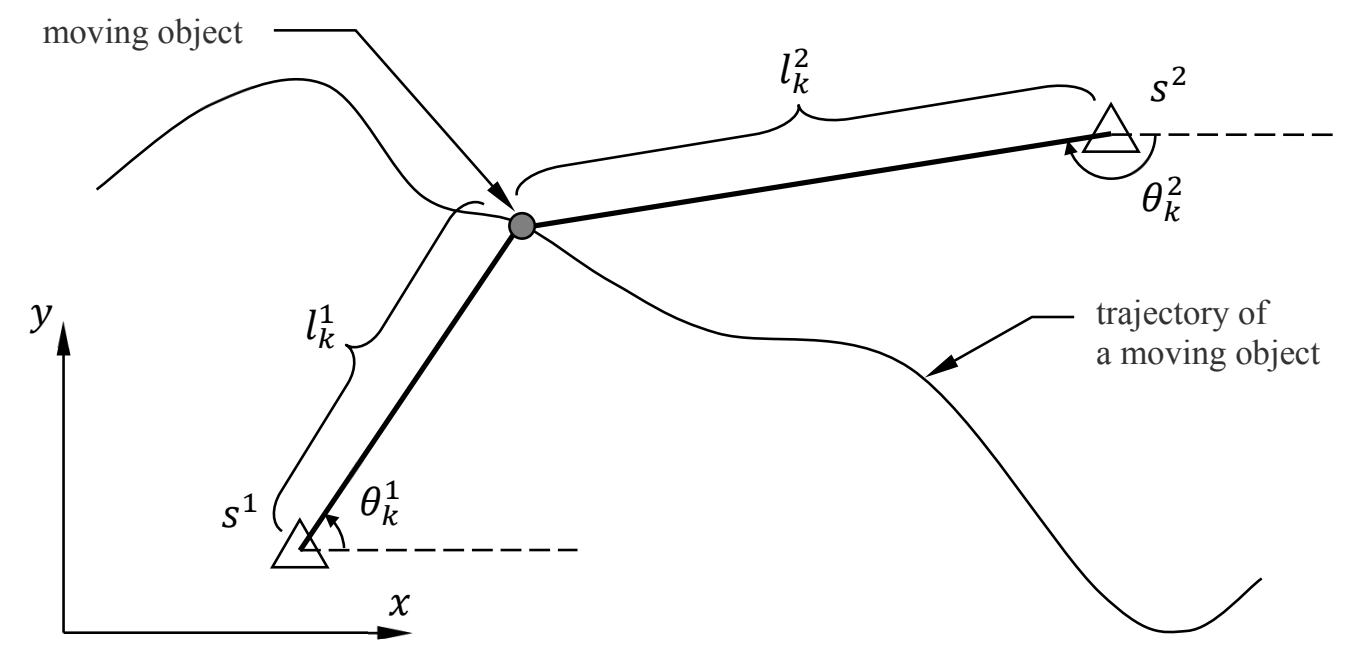

Fig. 2. The trajectory of a moving object observed from two fixed positions using the measurement of two directions and distances

Therefore, a model of the observation for the two positions with coordinates $\left(s_{x}^{1}, s_{y}^{1}\right)=(-1,-2)$ and $\left(s_{x}^{2}, s_{y}^{2}\right)=(1,1)$ can be formulated by the expression:

$$
\boldsymbol{z}_{k}=\nabla h\left(\boldsymbol{x}_{k}\right)+\boldsymbol{R}_{k}, \text { where } h\left(\boldsymbol{x}_{k}\right)=\left[\begin{array}{c}
\theta_{k}^{1} \\
l_{k}^{1} \\
\theta_{k}^{2} \\
l_{k}^{2}
\end{array}\right]=\left[\begin{array}{c}
\arctan \left(\frac{y_{k}-s_{y}^{1}}{x_{k}-s_{x}^{1}}\right) \\
\sqrt{\left(x_{k}-s_{x}^{1}\right)^{2}+\left(y_{k}-s_{y}^{1}\right)^{2}} \\
\arctan \left(\frac{y_{k}-s_{y}^{2}}{x_{k}-s_{x}^{2}}\right) \\
\sqrt{\left(x_{k}-s_{x}^{2}\right)^{2}+\left(y_{k}-s_{y}^{2}\right)^{2}}
\end{array}\right]
$$

Assuming no correlation between the measurements, the covariance matrix of measurement errors $\boldsymbol{R}_{k}$ will be white noise with expected value $E\left[\boldsymbol{r}_{k}\right]=0$ and $\sigma_{r}=0.05(\mathrm{rad})$, and it will take the following form:

$$
\boldsymbol{R}_{k}=\left[\begin{array}{cccc}
\sigma_{r}^{2} & 0 & 0 & 0 \\
0 & \sigma_{r}^{2} & 0 & 0 \\
0 & 0 & \sigma_{r}^{2} & 0 \\
0 & 0 & 0 & \sigma_{r}^{2}
\end{array}\right]=\left[\begin{array}{cccc}
0.0025 & 0 & 0 & 0 \\
0 & 0.0025 & 0 & 0 \\
0 & 0 & 0.0025 & 0 \\
0 & 0 & 0 & 0.0025
\end{array}\right]
$$

Taking partial derivatives from expression (12), the Jacobian matrix can be written as: 


$$
\boldsymbol{H}_{k}=\left[\begin{array}{cccc}
\frac{-\left(y_{k}-s_{y}^{1}\right)}{\left(x_{k}-s_{x}^{1}\right)^{2}+\left(y_{k}-s_{y}^{1}\right)^{2}} & 0 & \frac{x_{k}-s_{x}^{1}}{\left(x_{k}-s_{x}^{1}\right)^{2}+\left(y_{k}-s_{y}^{1}\right)^{2}} & 0 \\
\frac{x_{k}-s_{x}^{1}}{\sqrt{\left(x_{k}-s_{x}^{1}\right)^{2}+\left(y_{k}-s_{y}^{1}\right)^{2}}} & 0 & \frac{y_{k}-s_{y}^{1}}{\sqrt{\left(x_{k}-s_{x}^{1}\right)^{2}+\left(y_{k}-s_{y}^{1}\right)^{2}}} & 0 \\
\frac{-\left(y_{k}-s_{y}^{2}\right)}{\left.x_{k}-s_{x}^{2}\right)^{2}+\left(y_{k}-s_{y}^{2}\right)^{2}} & 0 & \frac{x_{k}-s_{x}^{2}}{\left(x_{k}-s_{x}^{2}\right)^{2}+\left(y_{k}-s_{y}^{2}\right)^{2}} & 0 \\
\frac{x_{k}-s_{x}^{2}}{\sqrt{\left(x_{k}-s_{x}^{2}\right)^{2}+\left(y_{k}-s_{y}^{2}\right)^{2}}} & 0 & \frac{y_{k}-s_{y}^{2}}{\sqrt{\left(x_{k}-s_{x}^{2}\right)^{2}+\left(y_{k}-s_{y}^{2}\right)^{2}}} & 0
\end{array}\right]
$$

where the position coordinates are: $\left.\left(s_{x}^{1}, s_{y}^{1}\right)=(-1,-2)\right)$ and $\left(s_{x}^{2}, s_{y}^{2}\right)=(1,1)$.

Similarly to the first example 5.1, to ensure identical conditions of simulation, the covariance matrices of state errors of $\boldsymbol{Q}_{k-1}$ and of measurement errors $\boldsymbol{R}_{k}$ for the system under consideration in all tested filters have the same form. At the outset of the process of estimation in all the analyzed methods of filtration, the same a priori initialization parameters of state vector and covariance matrix were specified.

\section{Testing filter credibility methods}

In order to verify the correctness and quality of operation of the Kalman filter, appropriate tests, simulations and statistical analysis of the innovation process should be carried out (Candy 1987).

The vector of measuring residuals should be determined while calculating state estimates in the Kalman filter. It expresses the difference (residue) between the observations obtained from the measurement devices and their predicted values. The rest of the measurement as a form of realization of the stochastic process is called innovation. In order to build a Kalman filter so as to be optimal, the necessary and sufficient condition is normally distributed innovation with zero expected value and covariance matrix corresponding to the actual errors of innovation (Andersen and Moore 1979, Candy 1987). Analyzing the properties of innovations with the help of statistical tests, it can be inferred about the quality of assumed filtering algorithm. During the statistical surveys, it is assumed that innovation is an ergodic Gaussian process (Andersen and Moore 1979, Candy 1987), i.e. such a stationary process for which the statistical parameter values in a set of execution are equal to the values of these parameters of its implementation any time.

It is convenient to study the statistical properties of the whole vector of innovation using a test of credibility. During the test, a scalar standardized evaluation is determined on the ground of analysis of innovation vector (measurement residuals) $\boldsymbol{v}_{k}$ and its covariance matrix $\boldsymbol{S}_{k}$, which is received during the correction stage (updating the impact of measurement) of the Kalman filter. The evaluation is made in the range (window) of a finite length of time $w$ :

$$
\hat{\rho}_{i}=\sum_{k=i-w+1}^{i} \boldsymbol{v}_{k}^{T}\left(\boldsymbol{S}_{k}\right)^{-1} \boldsymbol{v}_{k} \quad \text { for } i \geq w
$$


The calculated value of $\hat{\rho}$ has a distribution $\chi_{f}^{2}$ with $f=r \times w$ of freedom degrees, where $r$ is the size of the vector of innovation. Statistics $\hat{\rho}_{i}$ can be estimated with a confidence interval $\tau_{d}, \tau_{g}$, defined on the level of significance $\alpha=0.05$ for the distribution $\chi_{r \times w}^{2}$. In practice, this test determines the values in a sufficiently long time, and checks whether during approximately $95 \%$ of the time these values remain within the confidence interval, $\tau_{d}, \tau_{g}$, which confirms the proper operation of the Kalman filter. Delimitation of the confidence interval for estimator of variance coefficient $\sigma^{2}$ has the form:

$$
\tau_{d}, \tau_{g}=\frac{(n-r) \hat{\sigma}^{2}}{\chi_{d}^{2}} ; \frac{(n-r) \hat{\sigma}^{2}}{\chi_{g}^{2}}
$$

while the following statistics should be determined:

$$
P\left(\chi_{f}^{2} \geq \chi_{d}^{2}\right)=\frac{1+\gamma}{2}=\frac{2-\alpha}{2} \quad P\left(\chi_{f}^{2} \geq \chi_{g}^{2}\right)=\frac{1-\gamma}{2}=\frac{\alpha}{2}
$$

and the estimator of $\hat{\sigma}^{2}$ should be calculated:

$$
\hat{\sigma}^{2}=\frac{1}{n-w-r}\left[\sum_{i=1}^{n-w} \hat{\rho}_{i}^{2}-\frac{1}{n-w}\left(\sum_{i=1}^{n-w} \hat{\rho}_{i}\right)^{2}\right]
$$

In order to compare adjustment of the adopted filtration methods to the estimated dynamic system, the criterion of minimizing RMS error (Root Mean Square Error) for components (36) or (37) state vector for the entire computing session was used:

$$
\begin{gathered}
R M S\left(\boldsymbol{x}_{j}\right)=\sqrt{\frac{1}{n} \sum_{k=1}^{n}\left(\boldsymbol{x}_{j, k}-\hat{\boldsymbol{x}}_{j, k}\right)^{2}} \\
R M S(\boldsymbol{x})=\sqrt{\frac{1}{n \cdot p} \sum_{j=1}^{p} \sum_{k=1}^{n}\left(\boldsymbol{x}_{j, k}-\hat{\boldsymbol{x}}_{j, k}\right)^{2}}
\end{gathered}
$$

where:

$$
\begin{aligned}
& j \text { - number of the component of state vector } \boldsymbol{x}_{k}, \\
& n \text { - number of discrete computational epoch, } \\
& p \text { - size of state vector } \boldsymbol{x}_{k} .
\end{aligned}
$$

\section{The results of simulation tests}

The simulation results of a moving object which is observed from two fixed positions $\mathrm{s}^{1}$ and $\mathrm{s}^{2}$ using the measurement of directions $\theta_{k}^{1}$ and $\theta_{k}^{2}$ for both cases of the 500 calculation epochs are shown in figures 3 and 4 on the $x y$-plane trajectories for the tested filtration EKF, UKF, CDKF methods along with the actual trajectory of the object. The results of calculations are presented in and aggregate form for all tested 
methods of EKF, UKF, CDKF filtration respectively for case 1 (Fig. 3a) as well as case 2 (Fig. 4a). In order to increase the readability of the results for different methods, drawings have been enlarged for case 1 (Fig. 3b, 3c, 3d) and case 2 (Fig. $4 b, 4 c, 4 d)$.

However, the simulation results concerning the comparison of the real distance to the moving object from the position $s^{1}$ and its estimate are presented in aggregate form for all tested methods of EKF, UKF, CDKF filtration and EKS, UKS, CDKS smoothers, respectively for case 1 (Fig. 5a) and case 2 (Fig. 6a). In order to increase the readability of the results for different methods, drawings have been enlarged for case 1 (Fig. 5b, 5c, 5d) and case 2 (Fig. 6b, 6c, 6d).

On trajectory of maneuvering an object at turning points one could notice the decrease of accuracy estimated position (Fig. 3). The error growth is cause by assumed CWPV model, where the scalar amount approximates velocity. That situation leads to improper estimation of parameters of a moving object. The impact of this phenomenon may be decreased by extending the observation model with distance measurement (Fig. 4) or changing state model of dynamic system on continuous Wiener process acceleration (CWPA) model. Smoothers compared to the corresponding filtration methods in case 1 show higher efficiency of estimating the parameters which were omitted in the observation model (Fig. 5b, 5c, 5d). The results of estimating the distance for case 2 (Fig. 6) show a definite improvement in the sense of minimizing the RMS error both each filtering method and smoothers.

a)
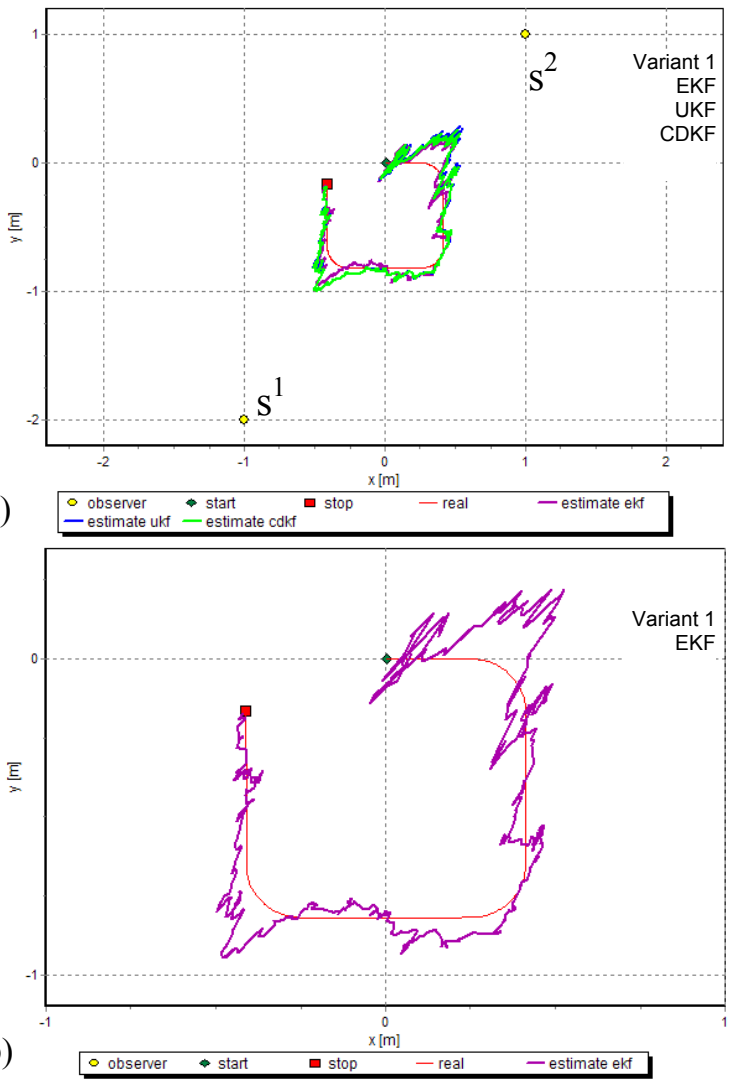
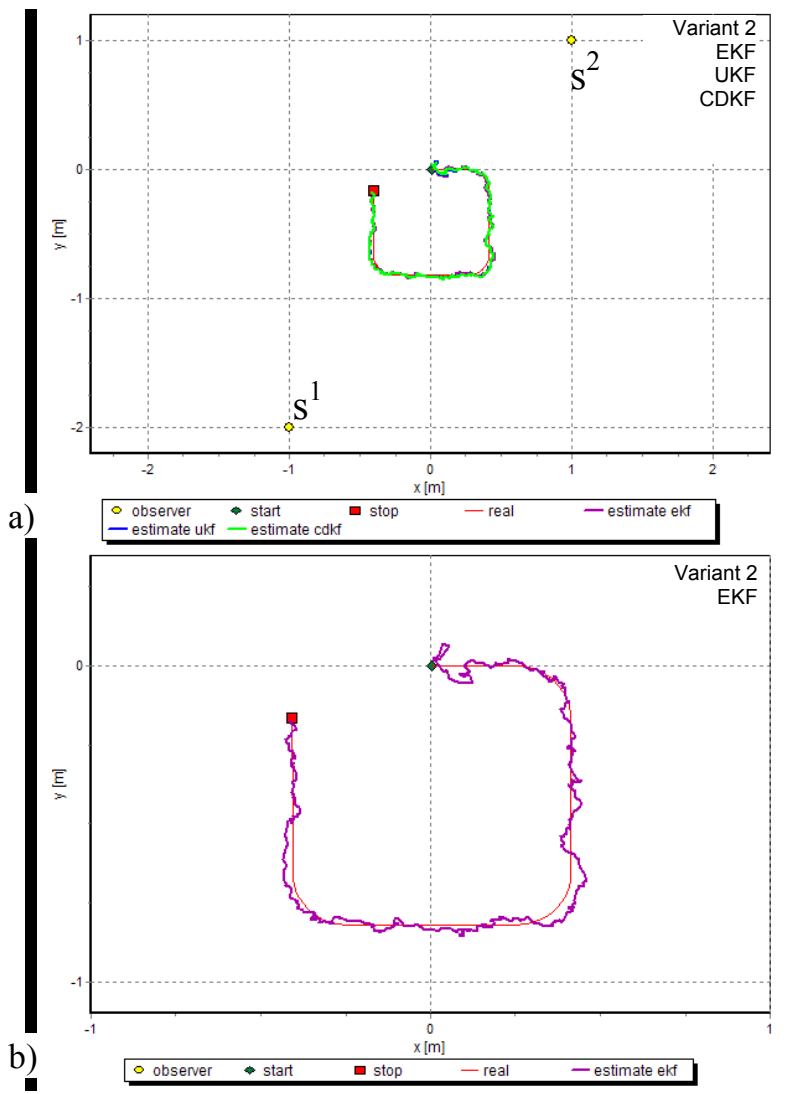
c)
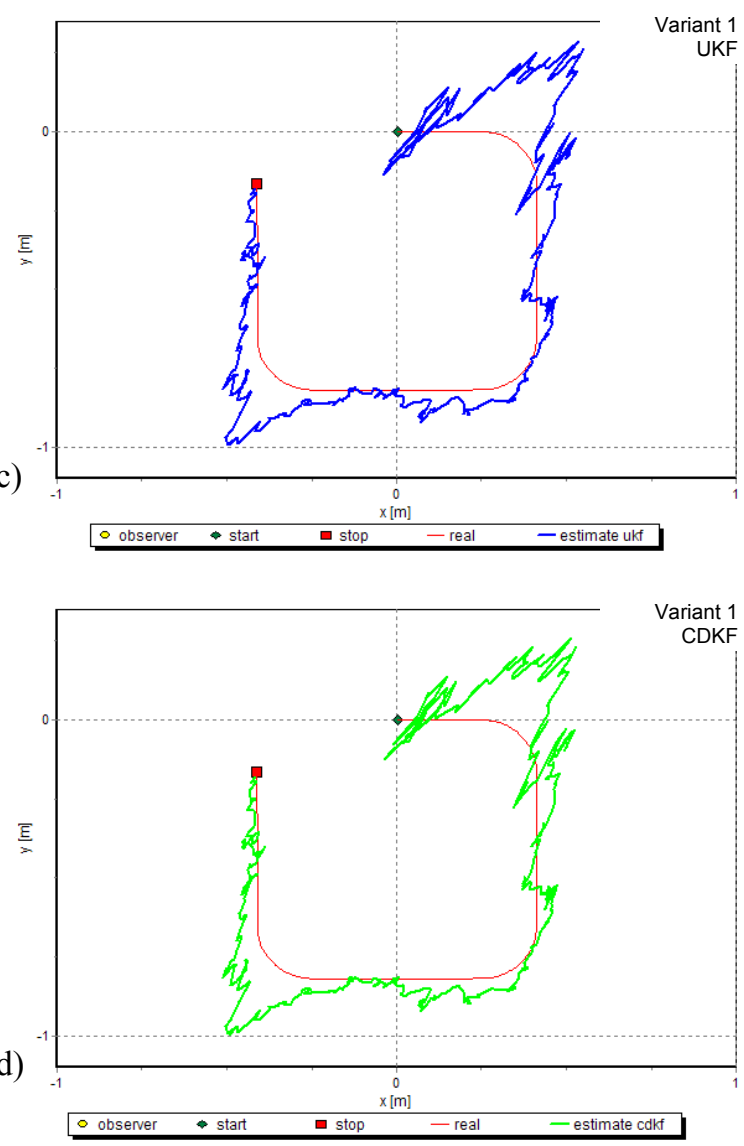

Fig. 3. The real trajectory and its estimates from observation points

$$
\mathrm{s}^{1}, \mathrm{~s}^{2} \text { in case } 1 \text { for: }
$$

a) all tested filters, b) EKF filter,

c) UKF filter, d) CDKF filter

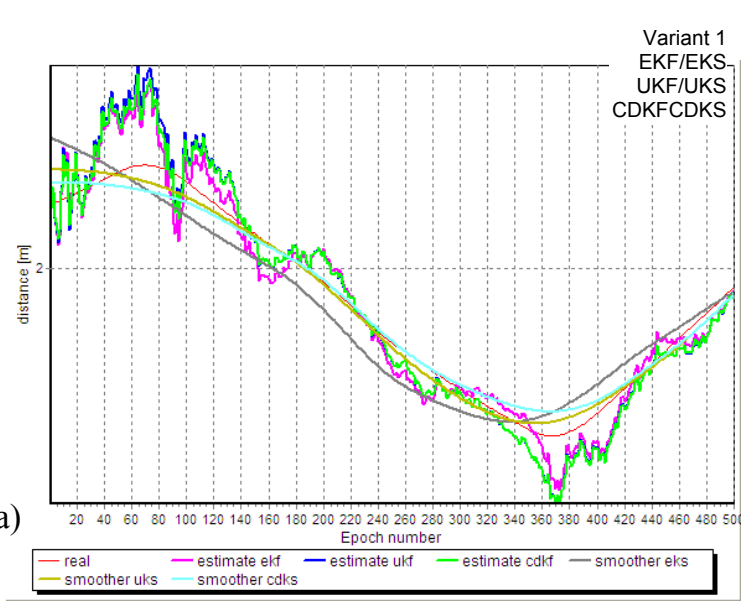

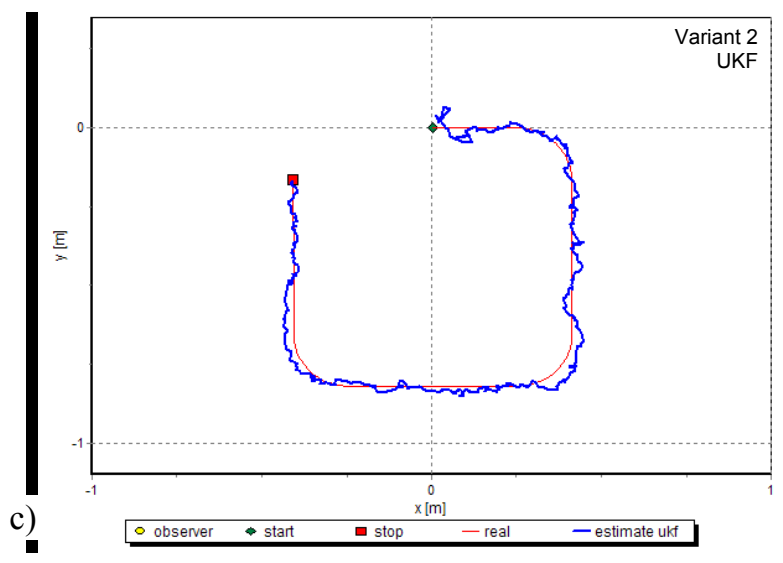

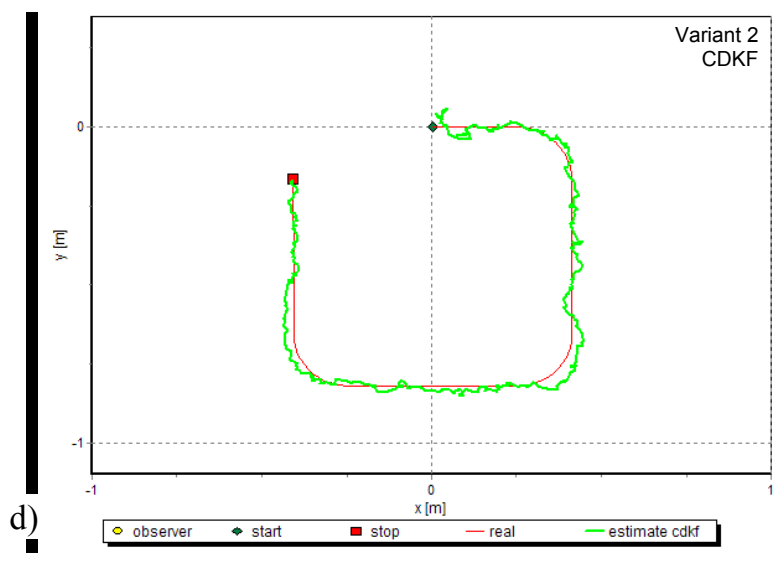

Fig. 4. The real trajectory and its estimates from observation points $s^{1}, s^{2}$ in case 2 for: a) all tested filters, b) EKF filter,

c) UKF filter, d) CDKF filter

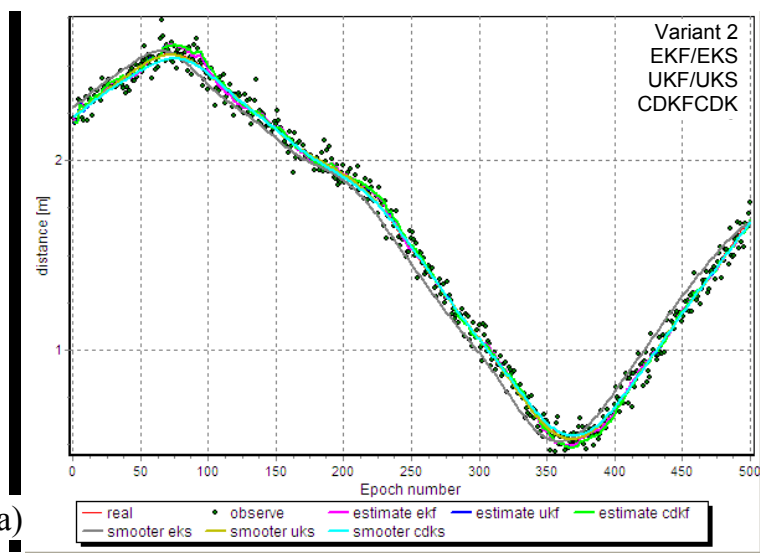


b)

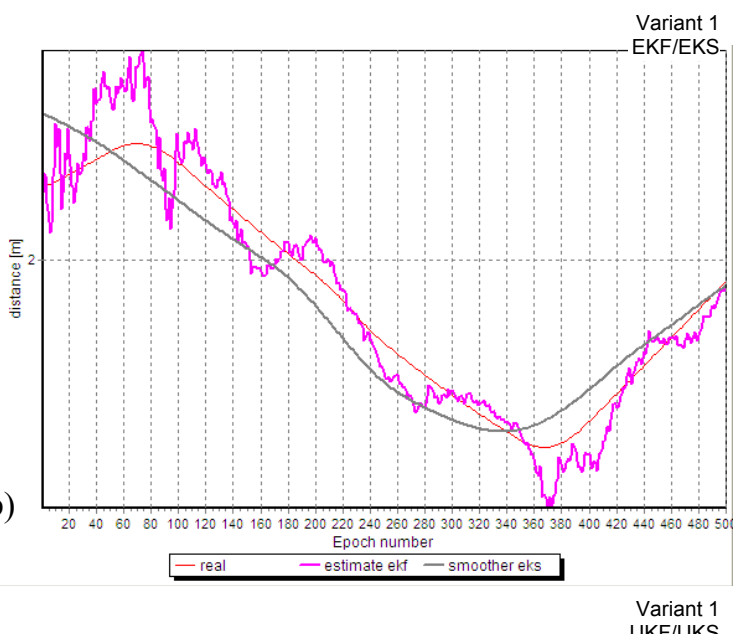

c)

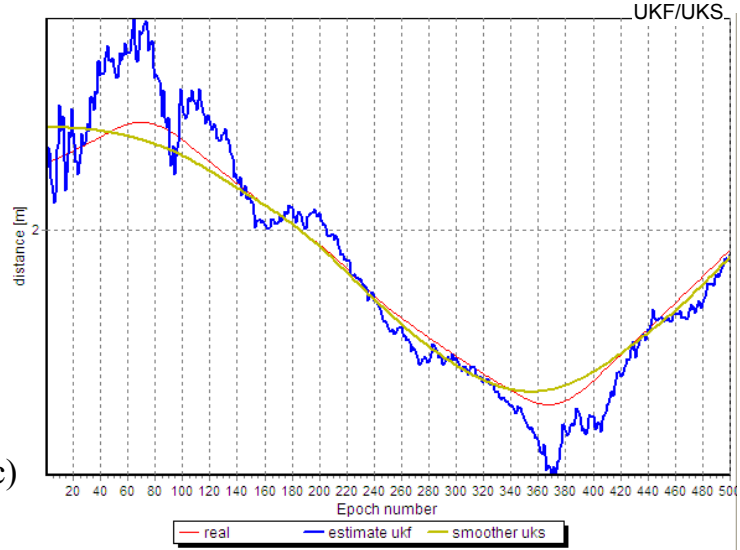

Variant 1

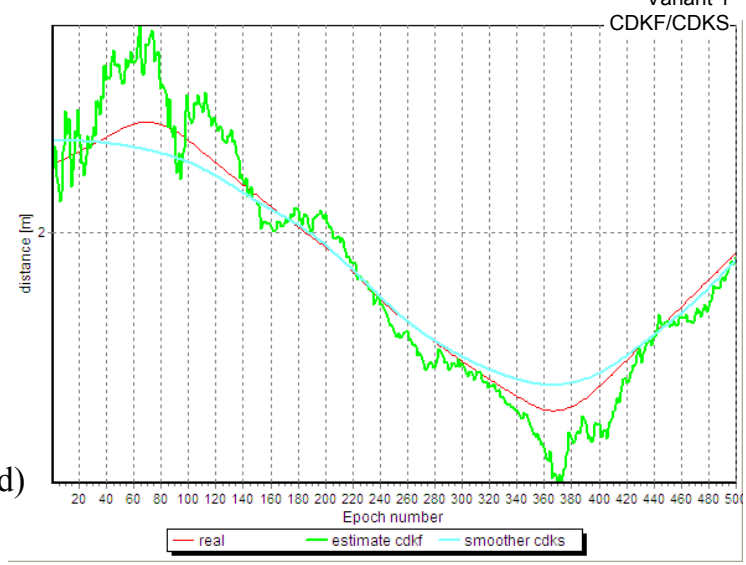

Fig.5. The real distance and estimates of its estimates from observation point $s^{1}$ in case 1 for:

a) all tested methods, b) EKF and EKS, c) UKF and UKS, d) CDKF filter and CDKS smoother
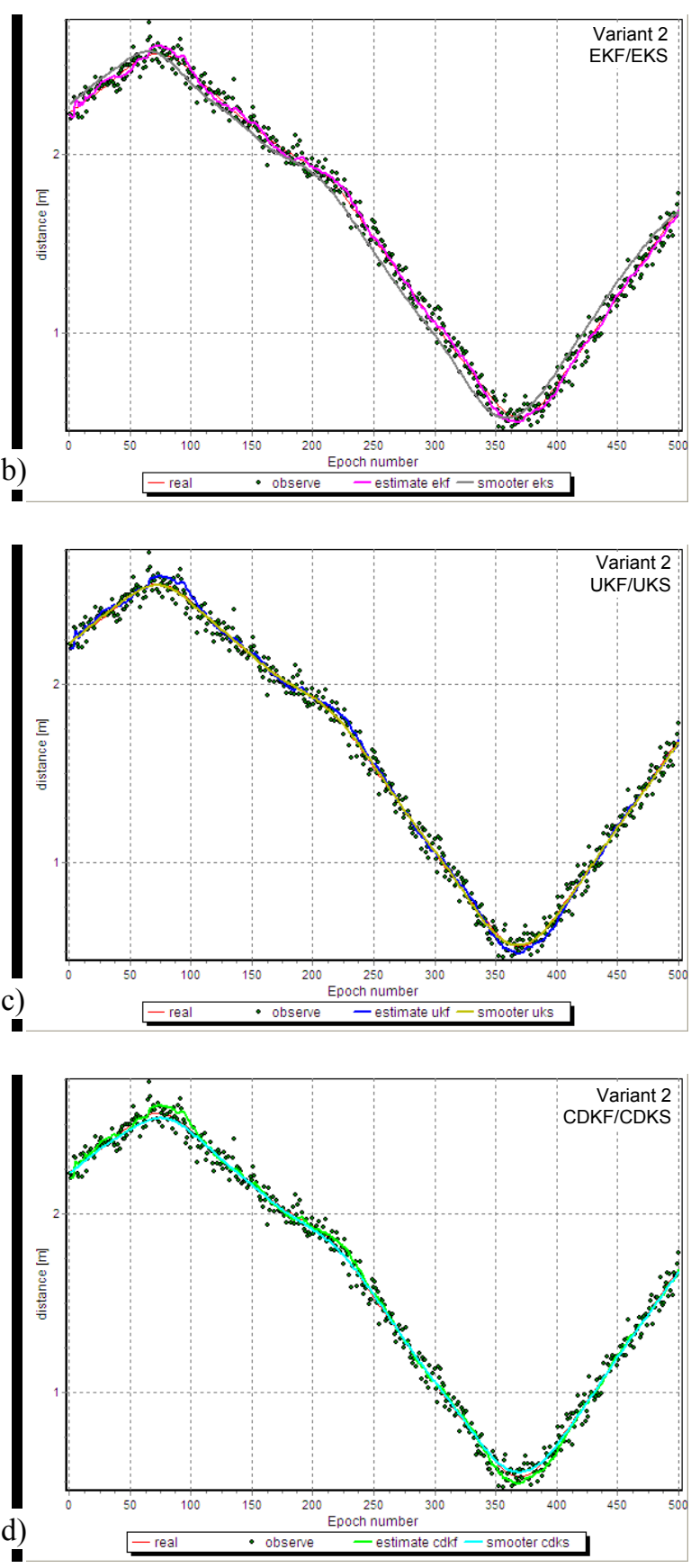

Fig.6. The real distance, estimates of its estimates with measurements from observation point $s^{1}$ in case 2 for:

a) all tested methods, b) EKF and EKS,

c) UKF and UKS, d) CDKF and CDKS 
$5.43 \% \hat{\rho}_{i}$ outside $\left\langle\tau_{d}, \tau_{g}\right\rangle=\langle 60.13,112.18\rangle$

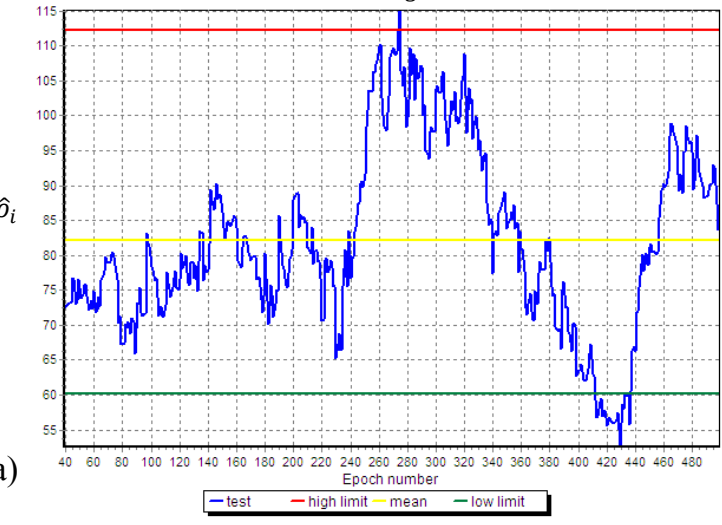

$5.43 \% \hat{\rho}_{i}$ outside $\left\langle\tau_{d}, \tau_{g}\right\rangle=\langle 59.72,111.41\rangle$

$\hat{\rho}_{i}$

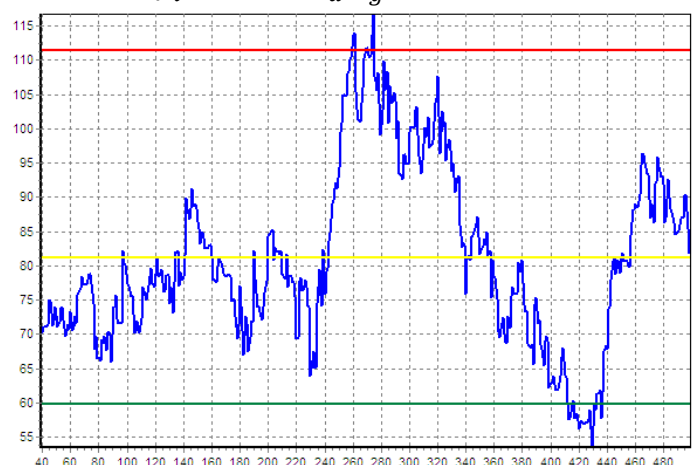

b)

$$
\text { -test } \quad \text { - high limit }- \text { mean } \quad-\text { low linit }
$$

$5.00 \% \hat{\rho}_{i}$ outside $\left\langle\tau_{d}, \tau_{g}\right\rangle=\langle 59.69,111.36\rangle$

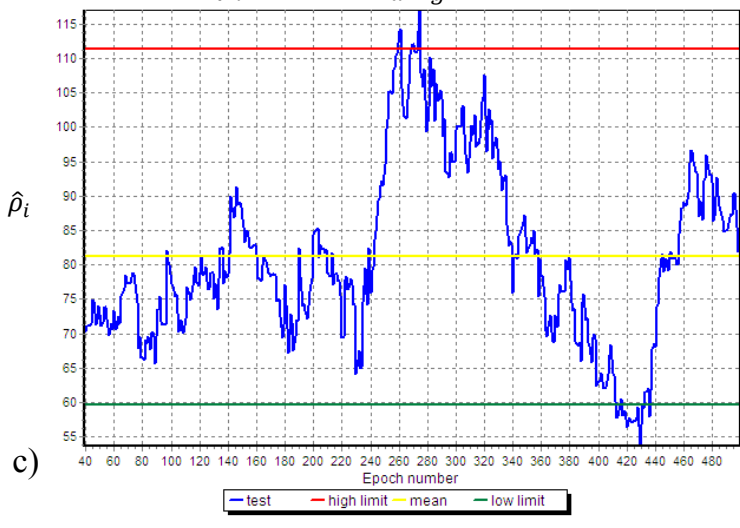

Fig.7. Test of scalar normalized evaluation of innovation in case 1 for window time $\mathrm{w}=40$ for:

a) EKF filter, b) UKF filter, c) CDKF filter
$3.70 \% \hat{\rho}_{i}$ outside $\left\langle\tau_{d}, \tau_{g}\right\rangle=\langle 58.28,108.74\rangle$

a)

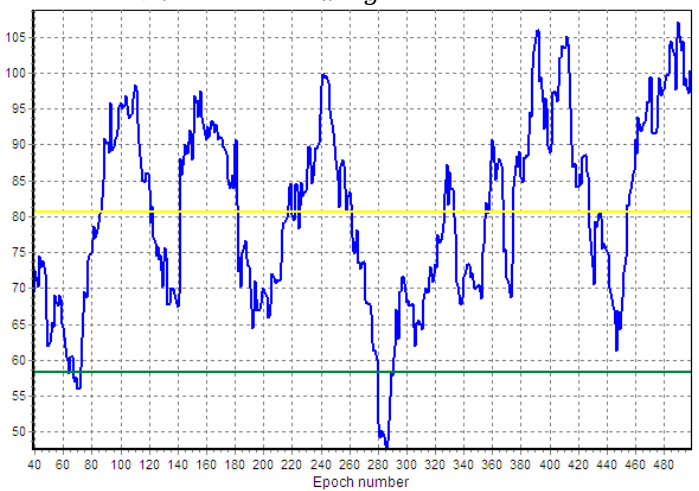

$3.26 \% \hat{\rho}_{i}$ outside $\left\langle\tau_{d}, \tau_{q}\right\rangle=\langle 67.76,126.42\rangle$

b)

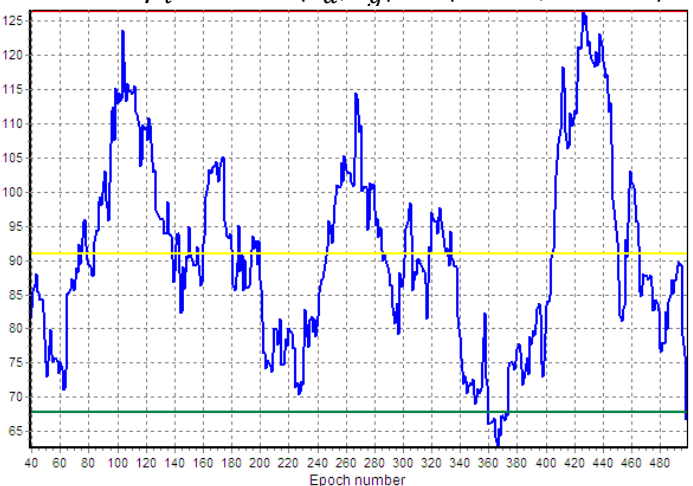

$3.26 \% \hat{\rho}_{i}$ outside $\left\langle\tau_{d}, \tau_{g}\right\rangle=\langle 69.14,128.99\rangle$

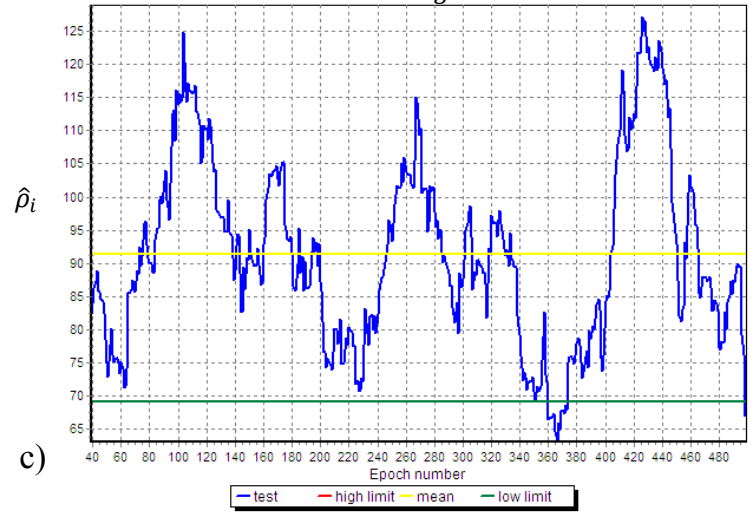

Fig.8. Test of scalar normalized evaluation of innovation in case 2 for window time $w=40$ for:

a) EKF filter, b) UKF filter, c) CDKF filter

The results of $\hat{\rho}_{i}$ test with time window of 40 epochs of entire innovation vector executed for the tested methods of EKF, UKF, CDKF filtration were shown in figures 7 and 8 . In each figure, information about limits of confidence range $\tau_{d}, \tau_{g}$ and the percentage share of the test outside the accepted confidence range was placed. The results presented in figures 7 and 8 proved that the work of designed filtration methods in conditions of matching to estimated process is correct.

The summary of variance and RMS mean square error for all simulation studies for the vector estimation of the vector for the position and velocity is provided 
respectively in (Table 1,2). The illustration of these tables are charts presented in (Fig. 9, 10). The results presented there for CDKF and CDKS methods, with a slight advantage over UKF and UKS, show them the best fit for the modeled state dynamic system. Moreover, the results achieved by each method of filtration in case 2 are more effective than the corresponding results of filtration methods in case 1 with the exception of the velocity vector and its component $\dot{y}_{k}$. Furthermore, the results achieved by UKS and CDKS smoothers have been better than the corresponding UKF and CDKF filtration methods (Fig. 9).

Table 1. Summary of RMS mean square error for position estimation in all simulation tests

\begin{tabular}{|c|c|c|c|c|}
\hline \multirow{2}{*}{ Case } & \multirow{2}{*}{ Method } & Vector of position & Component $x_{k}$ & Component $y_{k}$ \\
\cline { 3 - 5 } & & RMS [m] & RMS [m] & RMS [m] \\
\hline 1 & EKF & 0.072 & 0.056 & 0.084 \\
\hline 1 & EKS & 0.076 & 0.060 & 0.089 \\
\hline 2 & EKF & 0.018 & 0.018 & 0.018 \\
\hline 2 & EKS & 0.032 & 0.033 & 0.031 \\
\hline 1 & UKF & 0.084 & 0.065 & 0.099 \\
\hline 1 & UKS & 0.028 & 0.023 & 0.032 \\
\hline 2 & UKF & 0.016 & 0.016 & 0.017 \\
\hline 2 & UKS & 0.007 & 0.007 & 0.007 \\
\hline 1 & CDKF & 0.078 & 0.061 & 0.092 \\
\hline 1 & CDKS & 0.034 & 0.025 & 0.041 \\
\hline 2 & CDKF & 0.016 & 0.016 & 0.017 \\
\hline 2 & CDKS & 0.007 & 0.007 & 0.008 \\
\hline
\end{tabular}

Table 2. Summary for RMS mean square error of estimated velocity in all simulation tests

\begin{tabular}{|c|c|c|c|c|}
\hline \multirow{2}{*}{ Case } & \multirow{2}{*}{ Method } & Vector of velocity & Component $\dot{x}_{k}$ & Component $\dot{y}_{k}$ \\
\cline { 3 - 5 } & & RMS [m/s] & RMS [m/s] & RMS [m/s] \\
\hline 1 & EKF & 0.496 & 0.373 & 0.595 \\
\hline 1 & EKS & 0.596 & 0.428 & 0.726 \\
\hline 2 & EKF & 0.497 & 0.357 & 0.606 \\
\hline 2 & EKS & 0.497 & 0.434 & 0.675 \\
\hline 1 & UKF & 0.505 & 0.423 & 0.576 \\
\hline 1 & UKS & 0.560 & 0.413 & 0.675 \\
\hline 2 & UKF & 0.546 & 0.404 & 0.657 \\
\hline 2 & UKS & 0.546 & 0.404 & 0.657 \\
\hline 1 & CDKF & 0.502 & 0.421 & 0.572 \\
\hline 1 & CDKS & 0.548 & 0.399 & 0.665 \\
\hline 2 & CDKF & 0.549 & 0.406 & 0.662 \\
\hline 2 & CDKS & 0.564 & 0.429 & 0.673 \\
\hline
\end{tabular}




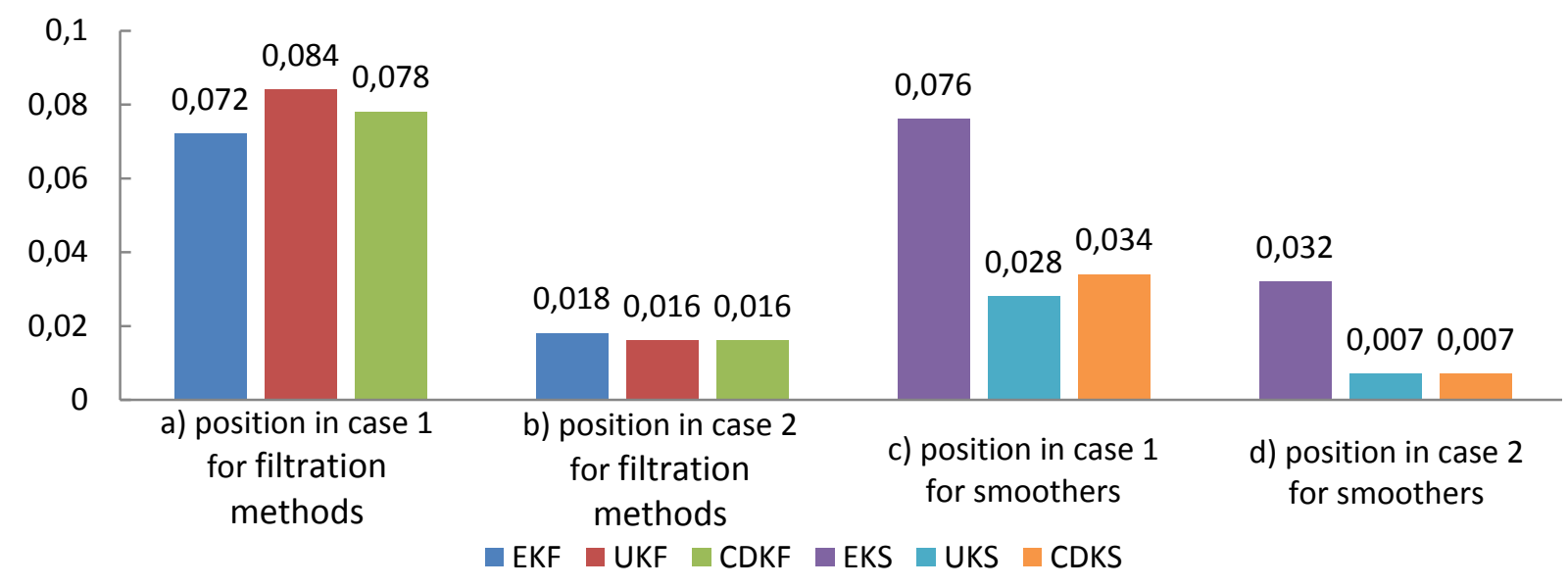

Fig.9. RMS mean square error of estimated position for all tested algorithms in both cases

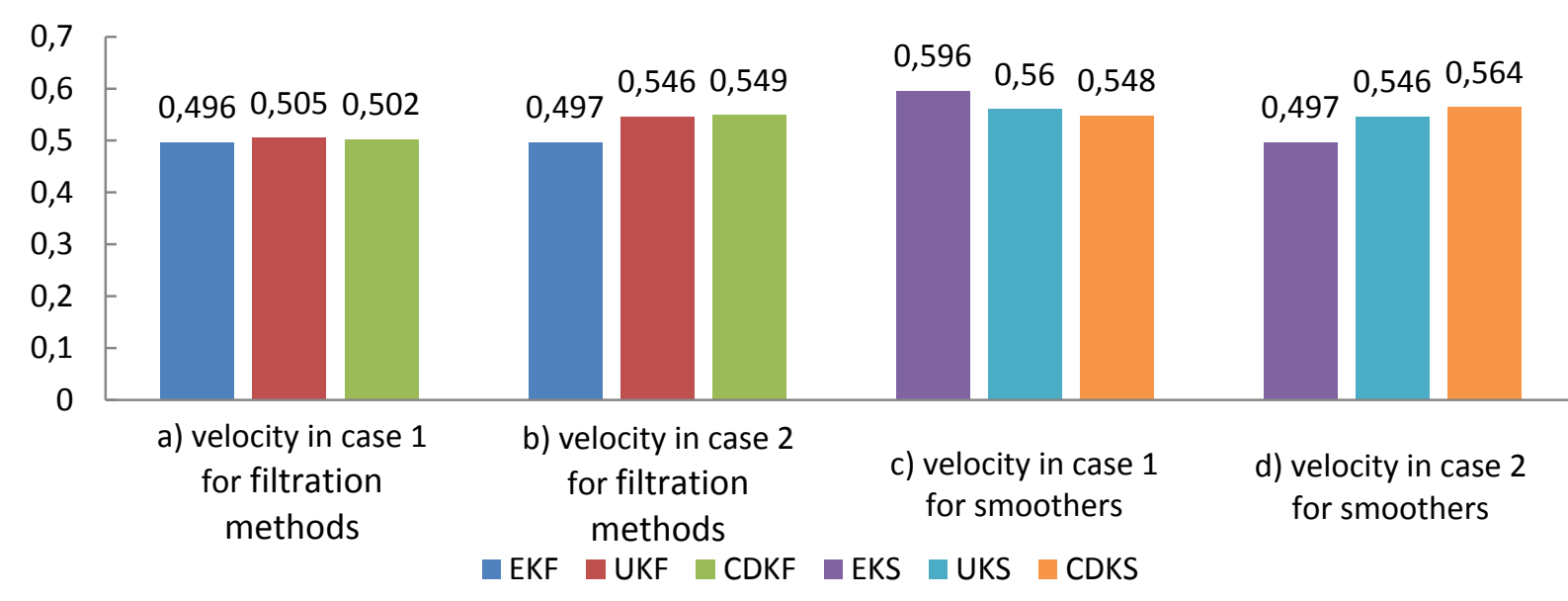

Fig.10. RMS mean square error of estimated velocity for all tested algorithms in both cases

The summary of variance and RMS mean square error of all the simulation tests for the direction and distance observed from fixed points $s^{1}$ and $s^{2}$ is shown in Table 3. The results presented there for each case of estimating the direction show a slight improvement in the sense of minimizing the RMS errors of UKF and CDKF methods compared to the EKF method (Fig. 11a, 11b). The improvement achieved for UKS and CDKS smoothers has been significant (Fig. 11c, 11d). The opposite of this situation are the results achieved by estimating the distance from two fixed positions where EKF shows the best performance among the methods of filtration (Fig. 12a, $12 b)$. The results obtained by the filtration methods in case 2 show a significant improvement compared to the corresponding results in case 1 (Fig. 12). The correctness of this model confirms the appropriateness of extending the observation model for distance measurement which was introduced in case 2. 
Table 3. Summary of RMS mean square error for estimated direction and distance

\begin{tabular}{|c|c|c|c|c|c|}
\hline Case & Method & $\begin{array}{c}\text { Direction } \\
\text { from position } \mathrm{s}^{1}\end{array}$ & $\begin{array}{c}\text { Distance } \\
\text { from position } \mathrm{s}^{1}\end{array}$ & $\begin{array}{c}\text { Direction } \\
\text { from position } \mathrm{s}^{2}\end{array}$ & $\begin{array}{c}\text { Distance } \\
\text { from position } \\
\mathrm{s}^{2}\end{array}$ \\
\cline { 3 - 6 } & $\begin{array}{c}\text { RMS } \\
{[\mathrm{rad}]}\end{array}$ & $\begin{array}{c}\text { RMS } \\
{[\mathrm{m}]}\end{array}$ & $\begin{array}{c}\text { RMS } \\
{[\mathrm{rad}]}\end{array}$ & $\begin{array}{c}\text { RMS } \\
{[\mathrm{m}]}\end{array}$ \\
\hline 1 & EKF & 0.014 & 0.098 & 0.013 & 0.099 \\
\hline 1 & EKS & 0.023 & 0.099 & 0.022 & 0.098 \\
\hline 2 & EKF & 0.012 & 0.015 & 0.012 & 0.014 \\
\hline 2 & EKS & 0.020 & 0.028 & 0.021 & 0.028 \\
\hline 1 & UKF & 0.013 & 0.116 & 0.012 & 0.117 \\
\hline 1 & UKS & 0.006 & 0.039 & 0.006 & 0.038 \\
\hline 2 & UKF & 0.010 & 0.015 & 0.010 & 0.014 \\
\hline 2 & UKS & 0.004 & 0.007 & 0.004 & 0.006 \\
\hline 1 & CDKF & 0.013 & 0.108 & 0.012 & 0.109 \\
\hline 1 & CDKS & 0.006 & 0.046 & 0.006 & 0.046 \\
\hline 2 & CDKF & 0.010 & 0.014 & 0.010 & 0.014 \\
\hline 2 & CDKS & 0.005 & 0.007 & 0.004 & 0.007 \\
\hline
\end{tabular}

The charts presented in (Fig. 11, 12) are illustration of the results in Table 3. Adding the distance measurements of tracking an object from fixed points $s^{1}$ and $s^{2}$ to the observation model in case 2 had an influence on increasing the efficiency of estimation of trajectory direction for EKF by $10-14 \%$ and for CDKF and UKF by 1519\% (Fig. 11b).

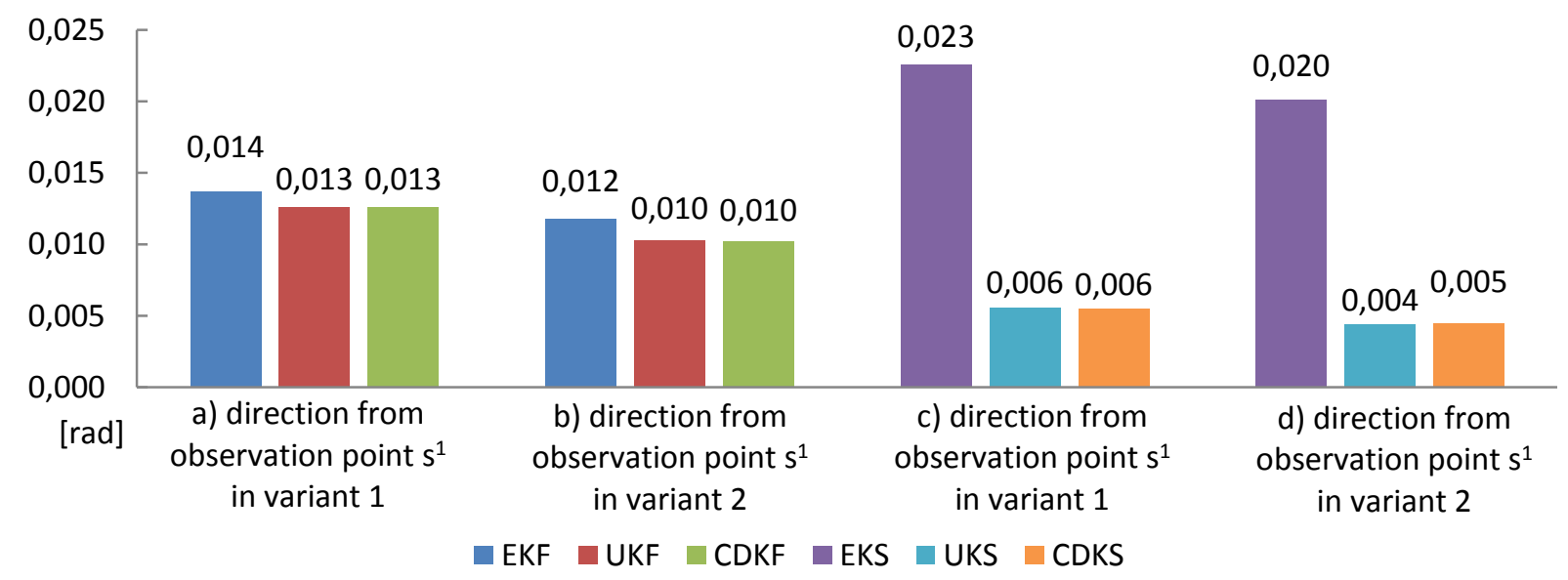

Fig.11. RMS mean square error of estimated direction for all tested algorithms in both cases 
Modification of the observation model in case 2 involved an increase in the efficiency of distance estimation of a tracked object from fixed points $s^{1}$ and $s^{2}$ for EKF by ca. $85 \%$ and for CDKF and UKF by ca. $87 \%$ (Fig. 12a, 12b). The lack of measurement of the estimated parameter influences a lesser performance of distance estimation in case 1 for CDKF and UKF methods in comparison to EKF (Fig. 12a). That situation demonstrates a higher sensitivity of CDKF and UKF filters with estimation of the parameter which was omitted in measurements (Fig. 12a). In case 1 UKS and CDKS smoothers compared to the corresponding UKF and CDKF filtration methods point to a higher estimation efficiency of distance which was omitted in observation model by $67 \%$ and $57 \%$ respectively (Fig. 12a, 12c). Among the three presented smoothers, UKS is characterized by the best performance (Fig. 12c, 12d). CDKS and UKS smoothers in case 1 and 2 allowed an increase in estimation efficiency compared to the corresponding filtration methods by $55 \%$ and $59 \%$ respectively (Fig. 11, 12).

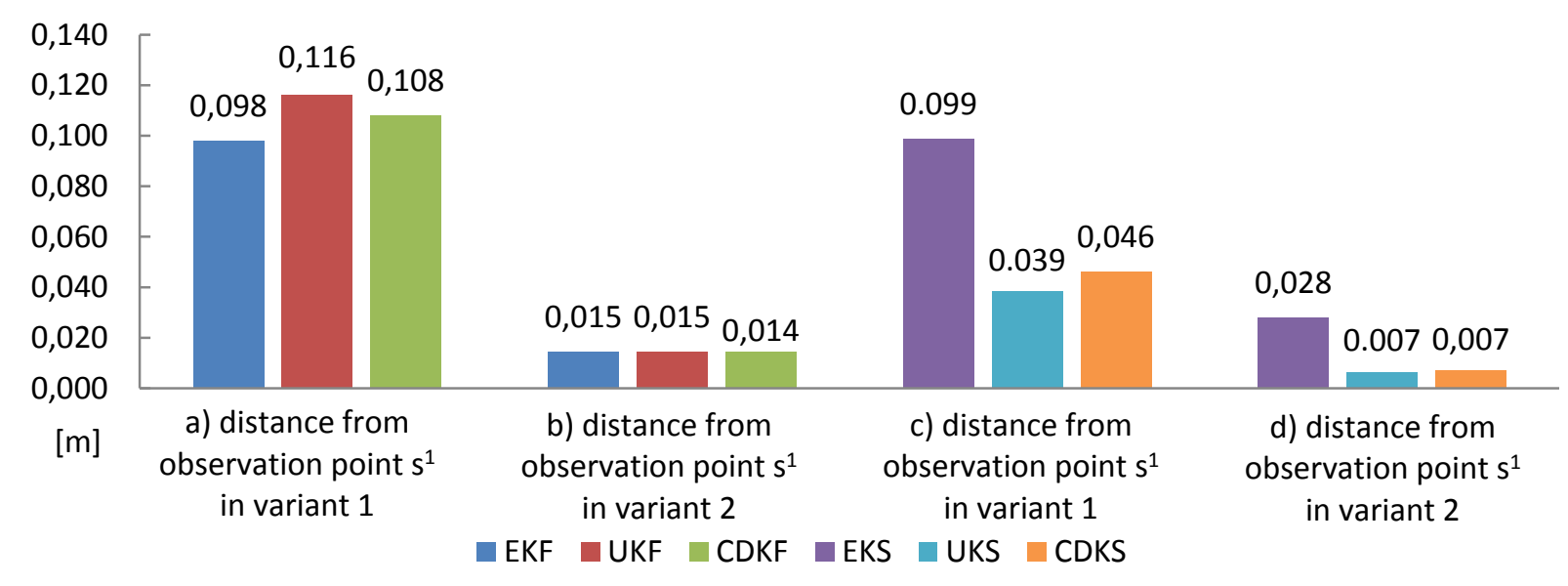

Fig.12. RMS mean square error of estimated distance for all tested algorithms in both cases

\section{Conclusions from the simulation studies}

The results of simulations performed for different algorithms allow drawing the conclusions. The filtering methods and smoothers implemented in the simulation examples work correctly in the conditions of matching to state of a dynamic system. The filtration methods used for the dynamics of CWPV models are the optimal estimators of the state of a dynamical system, if the actual working conditions correspond to the dynamics model. This is confirmed by statistical tests (Fig. 7, 8). Formulating the observation model (29) extended in comparison with (23) by measuring the distance has a significant impact on increasing the efficiency of filtration in the sense of minimizing the RMS error, which is also confirmed by comparative analysis of two pairs of drawings (Fig. 3) with (Fig. 4) and (Fig. 5) with (Fig. 6).

Simulation results of position and velocity estimation using variety filtering and smoothing methods have shown that UKF and UKS used as data processing algorithm give better accuracy of estimation in system with nonlinear dynamics than EKF and EKS. That performance is true but with some exceptions discussed in figure 9. Nonlinearity in observation model used in simulation is caused by polar 
transformation of coordinates. Such situation can be found very often in navigation. This shows UKF as more suitable for systems with strong nonlinearities than EKF. The amount of computations required by the CDKF and UKF is strongly higher than EKF, what makes it more demanding for computation units of integrated navigation systems.

The simulation examples helped to verify the correctness of work of filtration methods implemented in the BEA library. In the filtering models of the simulations the continuous Wiener process velocity (CWPV) model was used for a dynamic model. It would be interesting to apply the continuous Wiener process acceleration (CWPA) model instead of CWPV, what could lead to more efficient results in filtering methods. In the future work the accuracy of estimation could additionally be improved by using Particle Filtering method or filtering approaches with an interacting multiple model (IMM) algorithm as well.

\section{References}

Andersen, B. D. O., Moore, J. B. (1979). „Filtracja optymalna.” WNT, Warszawa, 1984. (oryg. Andersen B.D.O., Moore J. B. „Optimal filtering” Prentice-Hall Inc., Englewood Cliffs, New Jersey, USA, 1979).

Candy, J. V. (1987). „Signal Processing - The Model-Based Approch” McGraw-Hill, Singapore.

Christian, K. (2000). „Improvements of GNSS Receiver Performance Using Deeply Coupled INS measurements." ION GPS.

Grejner-Brzezinska, D. A., Toth C. K., and Yi Y. (2005) „On Improving Navigation Accuracy of GPS/INS Systems." Photogrammetric Engineering and Remote Sensing, Vol. 71, No. 4, 377-389.

Ito, K., Xiong, K. (2000). „Gaussian Filters for Nonlinear Filtering Problems.” IEEE Transactions on Automatic Control, 45(5), 910-927.

Kalman R. E., (1960) „A New Approach to Linear Filtering and Prediction Problems”, Trans. of the ASME - Journal of Basic Engineering, p. 35-45.

Kaniewski, P., (2006) „Aircraft Positioning with INS/GNSS Integrated System” Molecular and Quantum Acoustics, Vol. 27, p. 149-168.

Kim, H et al. (2003) „An Ultra Tightly coupled GPS/INS Integration using Federated Kalman Filter." ION GPS.

Knight, D. T. (1999). „Rapid Development of Tightly Coupled GPS/INS Systems.” Proceeding of ION International Meeting, Nashville, Tennessee.

Kwiecień, J., Malinowski, M., Bujnowski, S., Bujarkiewicz, B. (2006) „ATR TRACK III: The real-time GPS for public security.” Reports on Geodesy, No. 2(77), 179-185.

Konatowski, S. ; Sipa, T.(2004) „Position estimation using Unscented Kalman Filter” Annual of Navigation, No. 8, p. 97-110. 
Nørgaard M., Poulsen N., Ravn O., (1998) „Advances in Derivative-Free State Estimation for Nonlinear Systems", Technical Report IMM-REP-1998-15, Department of Mathematical Modelling, DTU, (revised Oct. 2004).

Nørgaard M., Poulsen N., Ravn O., (2000) „New Developments in State Estimation for Nonlinear Systems", Automatica, 36.

Rauch, H. E., Tung, F., Striebel, C. T., (1965) „Maximum likelihood estimates of linear dynamic systems". AIAA Journal, 3(8):1445-1450.

Rogers, R.M. (2007). „Applied Mathematics in Integrated Navigation Systems.” 3rd ed. Blacksburg, VA, USA: American Institute of Aeronautics and Astronautics, Inc.

Särkkä, S. (2006) „Recursive Bayesian Inference on Stochastic Differential Equations." Doctoral dissertation, Helsinki University of Technology Laboratory of Computational Engineering Publications Raport B54, Espoo.

Särkkä S., Vehtari A., and Lampinen J., (2007) „Prediction of ESTSP Competition Time Series by Unscented Kalman Filter and RTS Smoother", In Proceedings of ESTSP 2007, Espoo.

Särkkä, S., (2008) „Unscented Rauch-Tung-Striebel smoother”. IEEE Transactions on Automatic Control, 53(3):845-849.

Shin E, El-Sheimy N., (2005) „Backward Smoothing for Pipeline Surveying Applications" in Proceedings of ION NTM, pp. 921-927, U. S. Institute of Navigation, Fairfax VA, 24-26 January, San Diego CA.

Shin E., (2005) „Estimation Techniques for Low-Cost Inertial Navigation”, PhD Thesis, Department of Geomatics Engineering, University of Calgary, UCGE Report No. 20219, Canada.

van der Merwe, R., Wan, E.A. (2001) „Efficient Derivative-Free Kalman Filters for Online Learning." In Proc. of ESANN, Bruges.

van der Merwe, R., Wan, E.A. (2001) „The square-root unscented kalman filter for state and parameter-estimation." In Proceedings of the International Conference on Acoustics, Speech, and Signal Processing (ICASSP), Salt Lake City, Utah.

van der Merwe. R., Wan. E.A., Julier. S.J. (2004). „Sigma-Point Kalman Filters for Nonlinear Estimation and Sensor-Fusion:Applications to Integrated Navigation." In Proceedings of the AIAA Guidance, Navigation and Control Conference, Providence, RI.

Vorbrich, K.K., (2011) „Analysis of some low- and high-dynamics errors of Low-Cost IMU”, Geodesy and Cartography, Vol. 60, No 1, 2011, pp. 35-59. 


\section{Authors:}

Marcin Malinowski ${ }^{1)}$, marcinm@utp.edu.pl

Janusz Kwiecieńn ${ }^{2)}$, jkw@utp.edu.pl

1) Ph.D., Assistant Professor, Geomatics, Geodesy and Spatial Economy Department, University of Technology and Life Sciences, Kaliskiego street 7, 85-796 Bydgoszcz, Poland

2) Profesor, Chair of Geomatics, Geodesy and Spatial Economy Department, University of Technology and Life Sciences, Kaliskiego street 7, 85-796 Bydgoszcz, Poland 